\title{
Supra-Arcuate Ligament Blocks: Anatomy, Mechanisms, and Techniques
}

\author{
Huili Li (D)* \\ Jinghan Liang* \\ Peiqi Shao \\ Junwei Zheng \\ Rong Shi \\ Yun Wang (D)
}

Department of Anesthesiology, Beijing Chaoyang Hospital, Capital Medical University, Beijing, 100020, People's Republic of China

*These authors contributed equally to this work
Correspondence: Yun Wang Department of Anesthesiology, Beijing Chaoyang Hospital, Capital Medical University, No. 8, Gongtinan Road,

Chaoyang District, Beijing, 100020, People's Republic of China

Email wangyun129@ccmu.edu.cn

\begin{abstract}
Quadratus lumborum block (QLB) is clinically used for postoperative analgesia of abdominal or hip surgery, which can reduce the dose of general anesthetic, inhibit postoperative pain, and promote postoperative rehabilitation. However, accumulated studies have shown several drawbacks of QLB, such as only a small portion of local anesthetic spread into the thoracic paravertebral space, relatively longer onset time, and easilyinfluenced block effect by the integrity of lumbar fascia, etc. Therefore, on the basis of traditional anterior QLB, our group proposed the techniques of a supra-arcuate ligament block, which include a paramedian short-axis scanning approach, paramedian long-axis scanning approach, infra-lateral arcuate ligament QLB with the apposition zone between diaphragm and quadratus lumborum as drug diffusion target and supra-arcuate ligament block under direct laparoscopic visualization. Recent studies have demonstrated that the supra-arcuate ligament blocks have the advantages of clear anatomy, are easy to perform, and have rapid onset, which avoid some drawbacks of the conventional QLB techniques. This article reviews the anatomical basis, sonoanatomy, technical points, and clinical considerations of supra-arcuate ligament blocks.
\end{abstract}

Keywords: ultrasound guidance, arcuate ligament, quadratus lumborum block, thoracic paravertebral block

\section{Introduction}

Quadratus lumborum block (QLB) are widely used for postoperative analgesia of abdominal or hip surgery, which can reduce the dose of general anesthetic, inhibit postoperative pain, and promote postoperative rehabilitation. $^{1-3}$ Multiple approaches for QLB have been proposed, such as lateral, posterior, anterior, or intra-muscular QLB. ${ }^{4}$ With the wide use of QLB, obvious defects appear, such as the longer onset time, large volume of local anesthetic deposited in the lumbar fascia space, it being highly dependent on the ultrasound imaging quality, and it being easily-affected by the integrity of lumbar fascia, etc. Recently, our group proposed a novel anterior QLB at the supra-lateral arcuate ligament. ${ }^{5}$ Specifically, local anesthetic is injected directly anterior to the quadratus lumborum at the supralateral arcuate ligament under ultrasound guidance. This circumvents the barrier of arcuate ligament and makes it easier for local anesthetics to enter the thoracic paravertebral space. Based on that, we proposed several similar techniques, which are collectively called supra-arcuate ligament blocks. The supra-arcuate ligament block techniques avoid several drawbacks of conventional QLB, and provide a new choice for intraoperative and postoperative analgesia in abdominal or hip surgery. 


\section{Approaches and Working Principles of QLB}

\section{Different Approaches of QLB}

QLB block refers to the injection of local anesthetic through different approaches into either the fascial space around the quadratus lumborum or the quadratus lumborum to obtain regional anesthesia. The concept of QLB stems from the posterior transversus abdominis plane block. $^{6}$ ElBoghdadly et al divided QLB into lateral QLB (QLB1) and posterior QLB (QLB2) according to the needle tip position: QLB1 refers to needle entry from anteromedial to posterolateral. The final needle tip lies under the transversus abdominis aponeurosis, just anterolateral to the quadratus lumborum; the needle tip position of QLB2 lies in the lumbar interfascial triangle between the quadratus lumborum, latissimus dorsi, and erector spinae. Since then, increasing approaches, including transmuscular QLB, intramuscular QLB, and subcostal QLB, have been proposed. For transmuscular QLB, the needle tip lies in the fascial space between the psoas muscle and the quadratus lumborum. For subcostal QLB, the ultrasound probe scans in the paramedian sagittal plane, and the needle tip lies in the potential compartment between the quadratus lumborum and the transversalis abdominis anterior to it at L1-L2 level. ${ }^{7,8}$ Both transmuscular and subcostal QLB are regarded as anterior QLB. ${ }^{9}$ Because of the different QLB needle approaches, the range of local anesthetic spread varies significantly, leading to various block coverage and clinical effectiveness. ${ }^{10,11}$

\section{The Potential Communicating Pathway Between Lumbar Fascia Space and Subendothoracic Fascia Compartment - Important Anatomical Basis of QLB Working Principles}

The medial arcuate ligament spans from the L2 anterolateral margin to the L1 transverse process, while the lateral arcuate ligament spans from the $\mathrm{L} 1$ transverse process to the middle segment of the T12 rib. They together generate the low border of the diaphragm (Figure 1). The transversalis fascia covering the surface of the psoas major and quadratus lumborum muscles runs cephalad and split into two layers on arrival of medial and lateral arcuate ligament. One layer is fused with medial and lateral arcuate ligament and the other continues cephalad behind the arcuate ligament to be continuous with the endothoracic fascia. ${ }^{12,13}$ This anatomical feature constitutes a potential pathway for local anesthetic spread to the subendothoracic compartment with QLB approach, which is the region where the thoracic nerve roots are located.

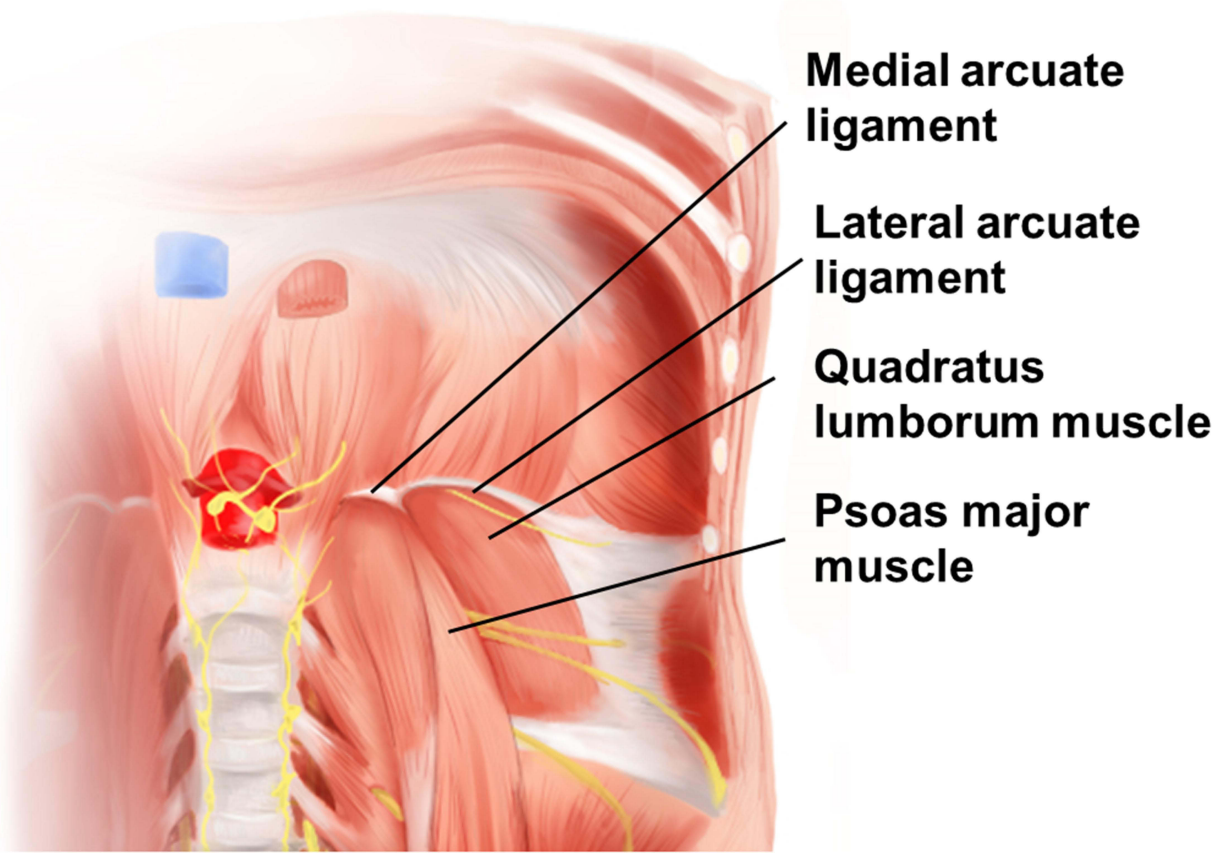

Figure I The anatomical relationship among quadratus lumborum, psoas muscle, and arcuate ligaments. The medial arcuate ligament spans from the $L 2$ anterolateral margin to the LI transverse process, while the lateral arcuate ligament spans from the LI transverse process to the middle segment of the TI 2 rib. The transversalis fascia travels cranially and splits into two layers on arrival of medial and lateral arcuate ligaments. The anterior layer continues with the medial and lateral arcuate ligament and the posterior layer continues cephalad with the endothoracic fascia. 


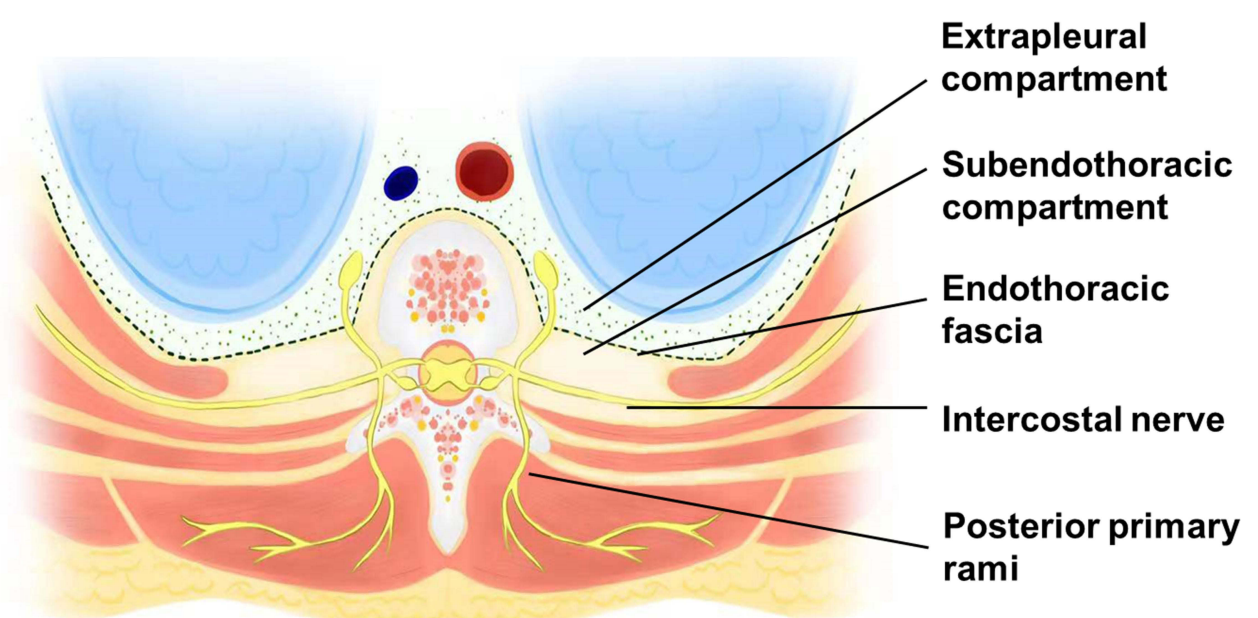

Figure 2 Relationship between the subendothoracic fascia compartment and the thoracic spinal nerves. Herein, the dashed line refers the endothoracic fascia, and its dorsal extraforaminal region is the subendothoracic fascia compartment, containing the ventral and dorsal rami of the thoracic spinal nerve roots. With the quadratus lumborum block, local anesthetic acts to block the thoracic spinal nerve roots by entering the subendothoracic fascia compartment, that is, into the thoracic space.

The QLB working principles are complex. One of the important principles for anterior QLB to obtain a higher blocking level is that local anesthetic diffuses from the fascia space around the quadratus lumborum through the posterior pathway of medial or lateral arcuate ligament of the diaphragm into the thoracic paravertebral space (Figure 2). Dam et $\mathrm{al}^{14}$ reported a cadaveric study. Through the transmuscular QLB approach, after administration of $30 \mathrm{~mL}$ solution containing the stain, dissection revealed that all cases had spread in the thoracic paravertebral space, up to the T9 level, and the thoracic sympathetic trunk and ventral rami of the spinal nerves in the corresponding paravertebral space were also stained. Elsharkawy et $\mathrm{al}^{15}$ found that the local anesthetic could reach the cadaveric thoracic paravertebral space in both QLB2 and subcostal QLB approaches, even reaching up to T6 level in the subcostal QLB approach. Additionally, local anesthetic may also spread to the thoracic paravertebral space through an intramuscular QLB approach. ${ }^{4}$ Therefore, local anesthetic diffuses cephalad and passes posterior to the arcuate ligament of the diaphragm into the thoracic paravertebral space to exert a blocking effect, which is an important working principle of QLB. ${ }^{14,16}$

\section{Development of Supra-Arcuate Ligament Block Techniques}

Although the conventional QLB has been favored by anesthesiologists over the last decade, several defects have begun to emerge in clinical practice: firstly, it takes a long time to get the blocking effect with anterior QLB, usually $20-30$ minutes. This is related to the time required for local anesthetic to spread from the fascia space around quadratus lumborum to the thoracic paravertebral space via the posterior pathway of arcuate ligaments. Secondly, the volume and dose of local anesthetic used is large. A considerable part of local anesthetic is deposited in the lumbar fascia space without fully reaching the effect site at the supra-arcuate ligament. There is a risk of local anesthetic poisoning during bilateral anterior QLB. Thirdly, the success rate of anterior QLB will be reduced when the imaging quality of fascia interspace structures is poor, which results in an unstable anesthetic effect. Finally, laparoscopic retroperitoneal organ surgery can destroy the integrity of the transversalis fascia, resulting in the leak of local anesthetic deposited in the lumbar fascia space and affecting the block quality.

Laterally, the quadratus lumborum muscles fill the space between T12 ribs and the iliac crest on both sides of the vertebral column. The lateral arcuate ligament spans from the L1 transverse process to the middle segment of the T12 rib. This indicates that the anterior compartment of quadratus lumborum muscle at the supra-lateral arcuate ligament is the critical relay for local anesthetics, since local anesthetics spread cephalad into thoracic paravertebral space through the arcuate ligament to exert its effect in QLB. Therefore, if local anesthetic is directly injected into the anterior of quadratus lumborum at the supra-lateral arcuate ligament, it will diffuse directly towards the low thoracic paravertebral space without overcoming the barrier of the 
medial and lateral arcuate ligament, and it does not store much in the lumbar fascial space below the arcuate ligament.

Further, our group thoroughly investigated the anatomical adjacency of the quadratus lumborum at the supra-lateral arcuate ligament. ${ }^{5}$ Two important anatomical characteristics should be highlighted. First, there is an apposition zone between quadratus lumborum and diaphragm (endothoracic fascia) above the lateral arcuate ligament level. Quadratus lumborum arises from the medial aspect of the low border of T12 rib and L1-L4 transverse processes, and ends at the superior border of iliac spine and the iliolumbar ligament. The lateral arcuate ligament at the low border of the diaphragm starts from L1 transverse process and ends at the mid portion of T12 rib across the quadratus lumborum. This suggests that there is an apposition zone between the quadratus lumborum and diaphragm above the lateral arcuate ligament level, which can be confirmed on the crosssectional CT image (Figure 3). Secondly, from the crosssectional CT image, the diaphragm and endothoracic fascia course towards the anterolateral margin of vertebral body above the lateral arcuate ligament level, while quadratus lumborum courses towards the facet joint or transverse processes of the vertebral body. This results in a triangular gap between diaphragm (endothoracic fascia) and quadratus lumborum, which opens on its basal side to the T12 paravertebral space (Figure 3). This is an important anatomical basis for the diffusion of local anesthetic to the lower thoracic paravertebral space through the lateral arcuate ligament in QLB. Based on the above two anatomical characteristics, we proposed two approaches to anterior QLB at the supralateral arcuate ligament under short or long axis scanning, respectively.

The anterior QLB at the supra-lateral arcuate ligament exhibit several advantages over the thoracic paravertebral block and conventional QLB that is widely used now. First, it can avoid the risk in lower thoracic paravertebral block, such as pneumothorax, Adamkiewicz artery injury, etc. Segmental arteries are an important source of blood supply to the spinal cord. They arise from deep carotid, intercostal, lumbar, and lateral sacral arteries, respectively. And their emanating radicular arteries enter the spinal canal via the intervertebral foramen and are mainly divided into the anterior and posterior radicular arteries. The thicker anterior root artery is called the Adamkiewicz

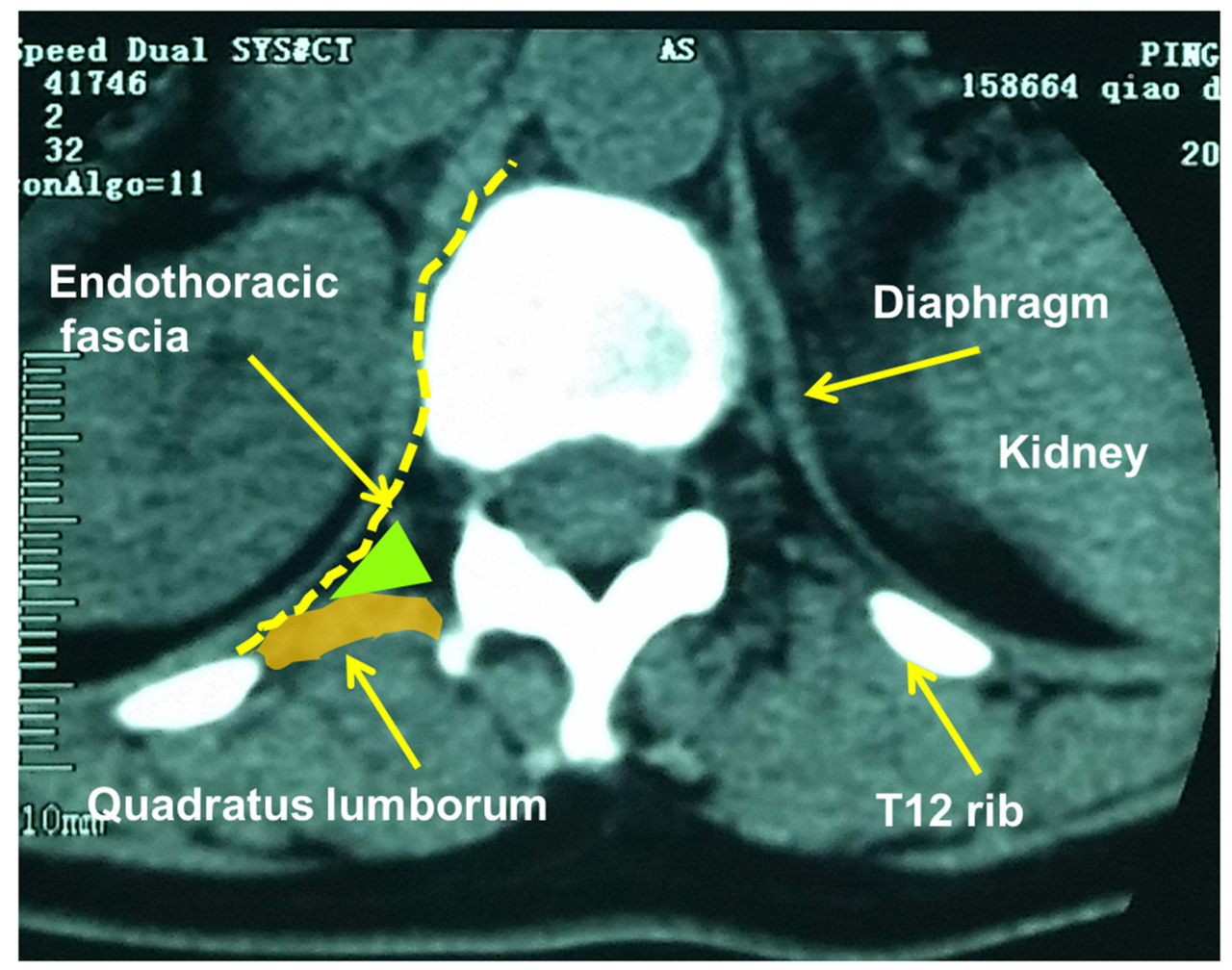

Figure 3 Two anatomical characteristics at the supra-lateral arcuate ligament were depicted in the cross-section computerized tomography. There was an apposition zone between the quadratus lumborum and diaphragm (endothoracic fascia) at the supra-lateral arcuate ligament. Diaphragm and endothoracic fascia course towards the anterolateral margin of the vertebral body at the supra-lateral arcuate ligament, while the quadratus lumborum courses towards the facet joint or transverse processes of the vertebral body. This results in a triangular gap between the diaphragm (endothoracic fascia) and quadratus lumborum (the green triangle refers to the gap). This triangular gap opens on its basal side to the TI2 paravertebral space. 
artery, also known as the root max artery. Its emanating position is mostly between T8-L3. The Adamkiewicz artery is the only feeding artery to the ventral aspect of the low thoracic and lumbosacral spinal cord. ${ }^{17}$ Injury of this artery by surgery or puncture can lead to spinal cord ischemic paraplegia. In this propsed innovative technique the puncture site is located on the apposition zone or the triangular zone between the quadratus lumborum and diaphragm (endothoracic fascia) above the lateral arcuate ligament level (a region of 5-6 cm lateral to the spinal midline), far from the paravertebral risky area at T8-L3, which can avoid puncture injury to the Adamkiewicz artery. Second, poor quality of structural imaging, such as transversalis fascia, reduces the QLB block success rate, leading to unstable block outcomes. ${ }^{18}$ The anterior QLB at the supra-lateral arcuate ligament is simple in anatomy, without complex fascial structures, and easier to achieve success. Third, laparoscopic surgery of retroperitoneal organs can disrupt the integrity of the transversalis fascia, resulting in the leak of local anesthetic stored within the lumbar fascia and reduced efficacy of the transmuscular QLB and subcostal QLB. ${ }^{19-21}$ In anterior QLB at the supra-lateral arcuate ligament, the puncture site is above the arcuate ligament level, directly into the lower thoracic paravertebral space. Local anesthetic may be less leaky caudally due to the arcuate ligament tension, while this remains to be confirmed by further studies.

\section{The Implementation of Supra-Arcuate Ligament Blocks}

There are multiple ways to implement supra-arcuate ligament blocks. Our group has proposed the anterior QLB at the supra-lateral arcuate ligament under a short or long axis scanning, the infra-arcuate QLB that takes the apposition zone between the diaphragm and quadratus lumborum as a drug diffusion target, and the supra-arcuate ligament block under direct laparoscopic visualization. These block techniques can be used for clinical anesthesia and postoperative analgesia in unilateral abdominal surgery, unilateral retroperitoneal cavity organ, as well as total abdominal surgery (bilateral block). Contraindications include coagulopathy, local infection by puncture, severe deformity of the spine, etc. A supra-arcuate ligament block under direct laparoscopic visualization can be used specifically for postoperative analgesia in laparoscopic retroperitoneal organ surgery.

\section{Ultrasound Guided Supra-Lateral Arcuate} Ligament Block: Paramedian Short-Axis Scanning Technique Patient Position and Scanning Method

Patients are usually taken in the lateral decubitus position with flexed knees and arched back. Then, the T12-L1 intervertebral space is located. A paramedian short axis oblique scan is performed with a low-frequency convex array ultrasound probe placed $3-4 \mathrm{~cm}$ lateral and perpendicular to the spine midline at the T12-L1 intervertebral space. The probe marking point is towards the lateral side and the ultrasound beam is slightly oblique toward the spine midline.

\section{Sonoanatomy}

A hyperechoic T12 costal bone surface is visible on the superolateral aspect of the ultrasound image, and there is an anechoic acoustic shadow below it. A typical hyperechoic bone surface curve is visible on the medial side, which consists of T12 vertebral body, the lamina (or facet joint), and the spinous processes from deep to shallow, respectively. A small depression between the vertebral body and the lamina (or facet joint) is defined as the T12-L1 extraforaminal region. Psoas muscle originates from T12 vertebral body, where the psoas muscle bundles are relatively weak and often not clearly identifiable in the transverse scanning. The anterolateral margin of the extraforaminal region is the diaphragm, which is obscured on breathing, and the kidney. When aspirated, the diaphragm moves down and shows a "double track sign". The dorsal aspect of T12-L1 paravertebral space is the podus-like quadratus lumborum and the transverse intersegmental ligament. In the lateral, diaphragm adherents to the quadratus lumborum muscle (apposition zone between diaphragm and quadratus lumborum muscle). Diaphragm courses medially to the anterolateral aspect of the vertebral body, while quadratus lumborum extends medially as intertransverse ligament and attaches to the ventral aspect of facet joints. The different rows of diaphragm and quadratus lumborum form a diaphragm-quadratus lumborum triangular gap, which is the puncture target area (Figure 4).

\section{Technical Points}

The in-plane puncture technique is generally used. Local infiltration anesthesia is performed at $0.5 \mathrm{~cm}$ from the low pole of the ultrasound probe $(2-3 \mathrm{~cm}$ from the spine midline). A $10 \mathrm{~cm}$ block needle is used for in-plane puncture. The needle tip direction is adjusted towards the diaphragm-quadratus lumborum triangular gap after the 


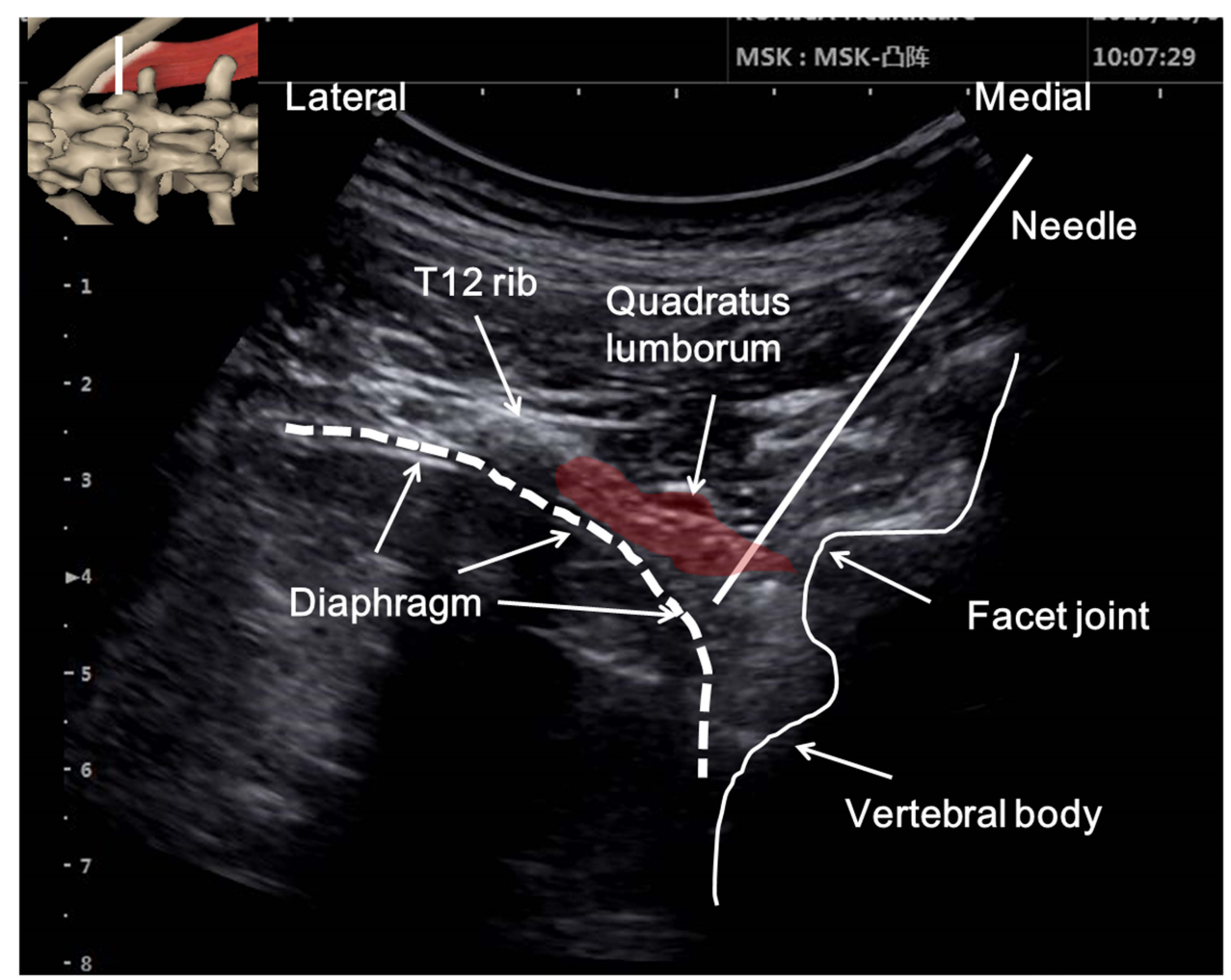

Figure 4 The transverse approach for an ultrasound-guided anterior quadratus lumborum block at the lateral supra-arcuate ligament. The sonogram shows the diaphragm apposition with the quadratus lumborum muscle and the triangular gap between them. The injectate target is the triangular gap between the quadratus lumborum and diaphragm. The embedded graph shows the site of the probe.

puncture needle is advanced into the erector spinae. The puncture needle breaks through the quadratus lumborum with a significant pop sensation. Between 15-20 mL local anesthetic can be given after the needle tip has reached the correct position confirmed by hydrodissection.

When the diaphragm is poorly identified, the patient can be instructed to breathe deeply to facilitate identification of the diaphragm on ultrasound images. Before puncture, it is important to know whether abnormal vessels are present in the target area in the color mode. The dermatomal coverage of sensory block can be detected between T6-7-L1-2 by ice cube method at 10 minutes after block. ${ }^{22}$ The associated complications include diaphragm injury, neuraxial injection, and abdominal visceral injury, etc.

\section{Ultrasound Guided Supra-Lateral Arcuate Ligament Block: Paramedian Long-Axis Scanning Technique \\ Patient Position and Scanning Method}

Patients are usually taken in the lateral decubitus position with flexed knees and arched back or the prone position if that is more stable. The low-frequency convex array probe is placed $6-8 \mathrm{~cm}$ lateral to the spine midline to perform the long-axis scan with the marking point of probe cephalad, identifying the quadratus lumborum cephalic attachment point on T12 rib. Then the ultrasound probe is moved medially towards the spine, till the acoustic shadow of the L1 transverse process tip is present on the sonogram. The probe is slid in the craniocaudal direction, imaging the area between the acoustic shadow of the T12 rib and the acoustic shadow of the L1 transverse process tip in the middle of the ultrasound sonogram. At this time a thin diaphragm running craniocaudally with respiration in the deep part of the quadratus lumborum is visible on ultrasound image. The ultrasound probe final scanning position is marked on the patient's skin.

\section{Sonoanatomy}

The hyperechoic T12 costal bone surface is visible on the cranial side, and there is an anechoic acoustic shadow below it. The diaphragm appears on an ultrasound as the hypoechoic muscles between the ventral as well as dorsal hyperechogenic bright lines, and moves craniocaudally 
with respiration. The deep part of the diaphragm is the kidneys. The diaphragm is visualized to extend caudally as thin transversalis fascia in the deep part of the quadratus lumborum when the ultrasound probe is moved slightly laterally. ${ }^{23}$ The low border of the diaphragm is the lateral arcuate ligament. The potential compartment between the quadratus lumborum and diaphragm (apposition zone) is the injection target area (Figure 5).

\section{Technical Points}

The in-plane puncture technique is generally used. The probe is placed in a pre-marked scanning position and locally adjusted to identify the T12 rib, L1 transverse process tip, as well as quadratus lumborum, and diaphragm between them. As the needle tip passes through the erector spinae and quadratus lumborum, it reaches the apposition zone between the diaphragm and quadratus lumborum. The puncture needle breaks through the quadratus lumborum with a significant pop sensation. Then $0.9 \%$ saline solution $(3 \mathrm{~mL})$ was administered through the pump tube and puncture needle to confirm the spread of fluid in the target space, which showed a downward depression of the diaphragm.

\section{Considerations}

When the diaphragm is poorly identified, the patient can be instructed to breathe deeply. The hyperechoic pleura is visible in the acoustic window caudal to the T12 rib. Before puncture, it is important to know whether abnormal vessels are present in the target area in the color mode. Under this scanning plane, the quadratus lumborum is strip-like and not easily discernible. Rudiments can first identify the thick quadratus lumborum by translating the probe laterally, then trace quadratus lumborum to this scanning plane. The key of this technique is the identification of lateral arcuate ligament at the low border of the diaphragm on ultrasound images. The diaphragm extends caudally as thin transversalis fascia in the deep part of the quadratus lumborum.

\section{Infra-Lateral Arcuate Ligament Block That Takes the Apposition Zone Between the Diaphragm and Quadratus Lumborum as a Drug Diffusion Target}

With the paramedian long-axis scan for the lateral supraarcuate ligament block, when the patient aspirates, the pleura can reach L1 transverse process in small part of

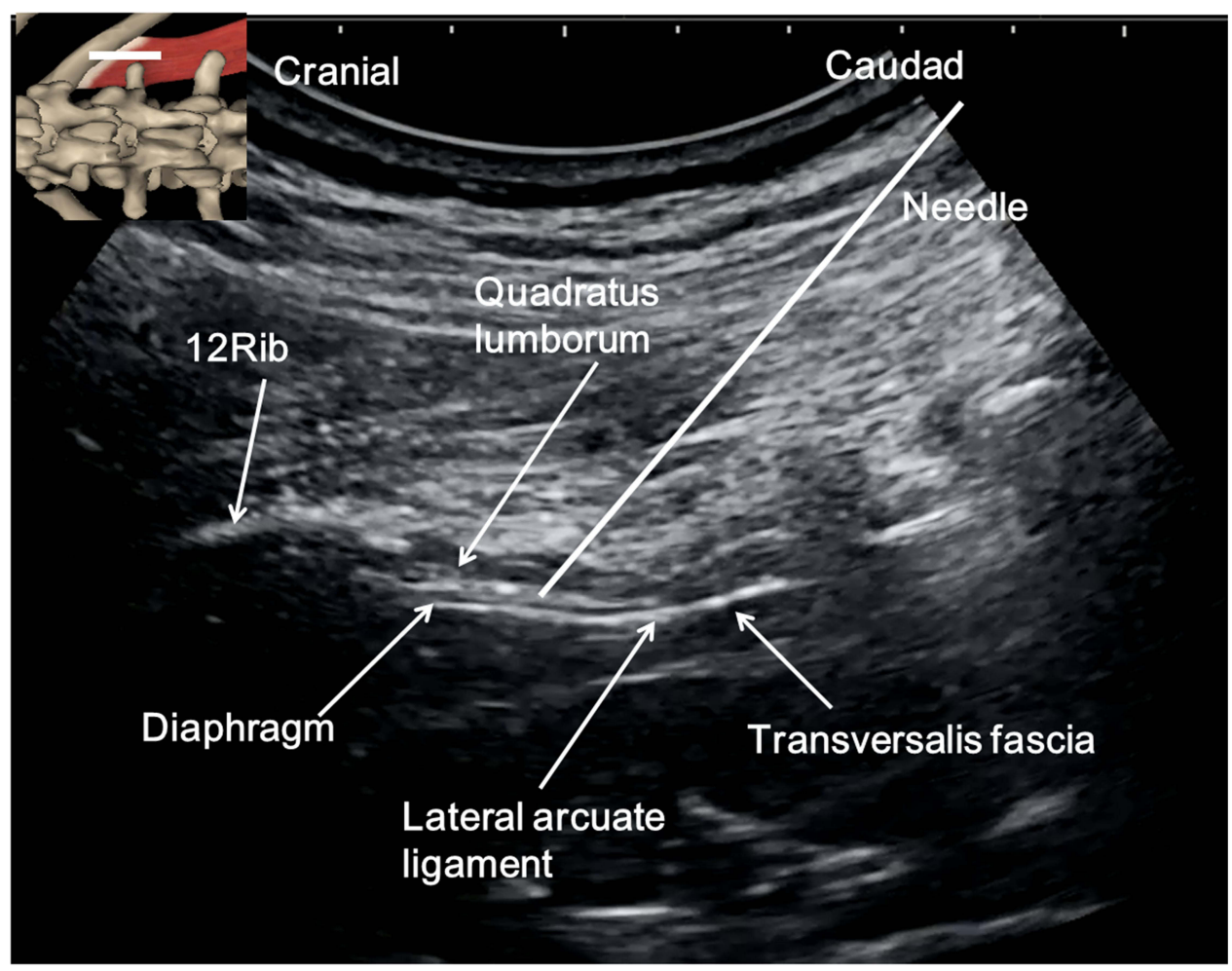

Figure 5 The parasagittal approach for ultrasound-guided anterior quadratus lumborum block at the lateral supra-arcuate ligament. The sonogram shows the apposition zone of the quadratus lumborum and diaphragm (endothoracic fascia). The lowest edge is the lateral arcuate ligament. The injectate target is the apposition zone between the quadratus lumborum and diaphragm (endothoracic fascia). The embedded graph shows the site of probe. 
patients. The lower pleural position may bring a risk of pneumothorax when performing the lateral supra-arcuate ligament block. Thus, the infra-lateral arcuate ligament block that takes the apposition zone between the diaphragm and quadratus lumborum as a drug diffusion target can be considered for these patients.

\section{Patient Position, Scanning Method, and Sonoanatomy} Patients are usually taken in the lateral decubitus position with flexed knees and arched back or the prone position that is more stable The scanning method and sonoanatomy are similar to those for the supra-lateral arcuate ligament block (Figure 6).

\section{Technical Points}

The in-plane puncture technique is generally used. The probe is placed in a pre-marked scanning position and locally adjusted to identify T12 rib, L1 transverse process tip, as well as quadratus lumborum and diaphragm between them. The ultrasound probe is slightly translated laterally until the L1 transverse process tip just disappears. Now, the diaphragm is visualized to extend caudally as thin transversalis fascia in the deep part of the quadratus lumborum on ultrasound images. The low border of the diaphragm is the lateral arcuate ligament. Then, an in-plane puncture is performed in the cranial-caudal direction. The target is the potential compartment between the quadratus lumborum and transversalis fascia, which is below and close to the lateral arcuate ligament. When the puncture needle passes through the erector spinae and quadratus lumborum to the target area, $0.9 \%$ saline solution $(3-5 \mathrm{~mL})$ is administered through the pump tube and puncture needle. After confirming the spread of fluid to the diaphragm-quadratus lumborum apposition zone above the lateral arcuate ligament, $20-30 \mathrm{~mL}$ local anesthetic is given. The diaphragm is observed to be downward depressed on the sonogram.

\section{Considerations}

The low border of the diaphragm is the lateral arcuate ligament. The puncture target of this technique is below the lateral arcuate ligament and away from the pleura, which can avoid pneumothorax complications. Elsharkawy et al proposed the technique of subcostal anterior QLB. The ultrasound probe is placed at L1-2 level for paramedian oblique-longitudinal scanning, where the diaphragmquadratus lumborum apposition zone is not visible on ultrasound images. In the infra-lateral arcuate ligament block that takes the apposition zone between the diaphragm and quadratus lumborum as a drug diffusion target, it is possible to

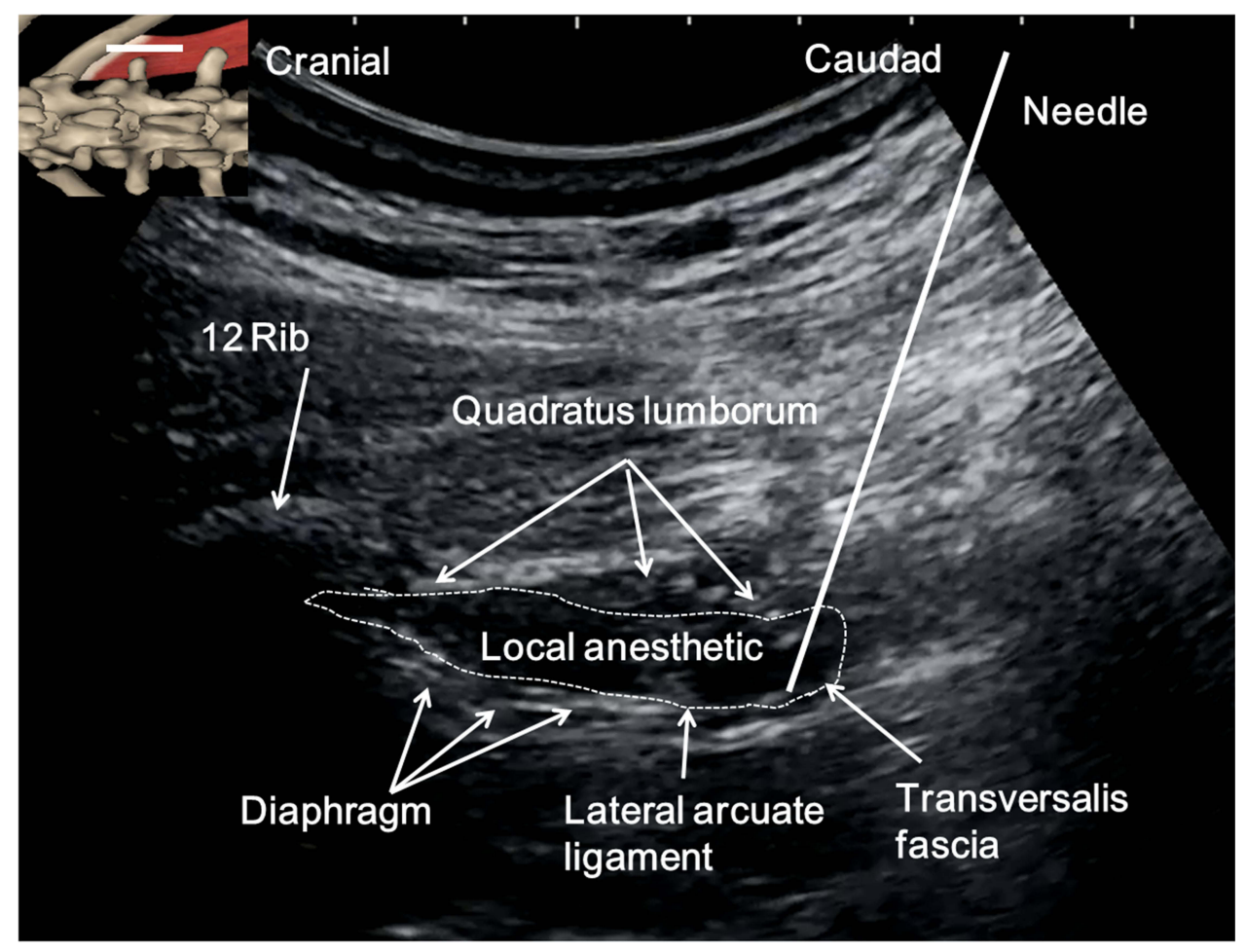

Figure 6 The infra-lateral arcuate ligament quadratus lumborum block with the apposition zone between the diaphragm and quadratus lumborum as a drug diffusion target. The sonograms showing the local anesthetic spread under paramedian sagittal view. The target injection site is below the lateral arcuate ligament and between the investing fascia of the quadratus lumborum and the transversalis fascia. Following the injection, an apparent downward displacement of the diaphragm can be observed. 
observe real-time diffusion of local anesthetic into the diaphragm-quadratus lumborum apposition zone after injection. This technique can also be seen as a modification of the subcostal anterior QLB.

\section{Supra-Arcuate Ligament Block Under Direct Laparoscopic Visualization}

Surgery on retroperitoneal organs under laparoscopy is widely carried out in the clinic, such as laparoscopic resection of renal, partial renal, or renal cysts, etc. When performing laparoscopic renal surgery, it is necessary to isolate and ligate the renal artery. Since the renal artery is located near the medial arcuate ligament, urologists usually go on to explore the renal artery by looking for the medial arcuate ligament of diaphragm crossing the surface of the psoas major muscle. ${ }^{19}$ The medial arcuate ligament arises at the anterolateral border of the L2 vertebral body, spans the psoas major muscle, and ends at the L1 transverse process. These anatomical relationships can be clearly revealed under direct laparoscopic visualization. In the upper and middle thoracic segments, the anterolateral boundary of thoracic paravertebral space is parietal pleura. Whereas, in the low thoracic paravertebral space (T10-T12), it is bounded anterolaterally by the diaphragm due to the presence of the apposition zone of the diaphragm. Hence, under direct laparoscopic visualization, local anesthetic can be injected through the medial arcuate ligament into the dorsal aspect of the diaphragm, that is, the lower thoracic paravertebral space, thereby acting as a lower thoracic paravertebral block (Figure 7). This technique can be performed by urologists under direct laparoscopic visualization and is simple as well as convenient (unpublished data). There are certain advantages of this technique for obese or elderly patients who have difficulty performing ultrasound-guided supra-arcuate ligament blocks or thoracic paravertebral blocks due to the poor quality of ultrasound images. ${ }^{24}$

\section{Patient Position}

The lateral or supine position is often used for laparoscopic retroperitoneal organ surgery.

\section{Technical Points}

According to the surgical habits of urologists and surgical demands, a supra-arcuate ligament block under direct laparoscopic visualization can be performed after the beginning of pneumoperitoneum, or before the ending of pneumoperitoneum at the completion of surgery. Taking the laparoscopic retroperitoneal nephrectomy under lateral position, for example, after establishing the pneumoperitoneum, urologists open the renal fascial sac, dissect the fascia tissues cephalad until finding the media arcuate ligament that spans the psoas major muscle on the cephalic surface of the psoas muscle. The medial arcuate ligament is located at the lowest border of the diaphragm and appears white. A $2 \mathrm{~cm}$ scalp needle with an extension tube is placed into the free space by manipulation forceps. The position, where the

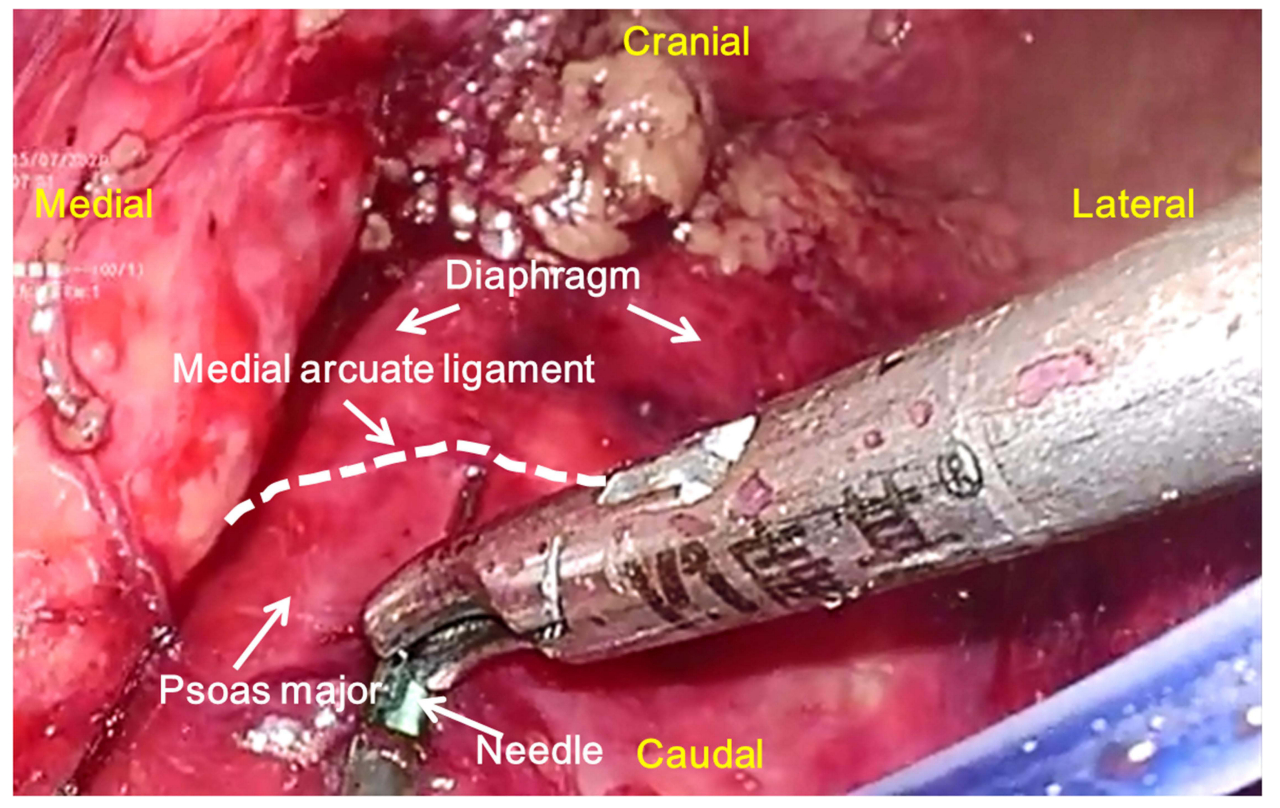

Figure 7 The diaphragm apposition zone block via the posterior pathway of medial arcuate ligament under direct laparoscopic visualization was performed. 
needle is clipped by manipulation forceps, is adjusted to make the scalp needle puncture cephalad from the lower border midpoint of the medial arcuate ligament to the dorsal aspect of the diaphragm, with the direction parallel to the spine midline and a 15 degree angle of needle to psoas muscle surface (Figure 8). After negative aspiration without blood or cerebrospinal fluid, 3$5 \mathrm{~mL} 0.9 \%$ saline solution is administered. The spread of liquid to the cephalic side is then observed. Then 20 $30 \mathrm{~mL}$ local anesthetic is administered.

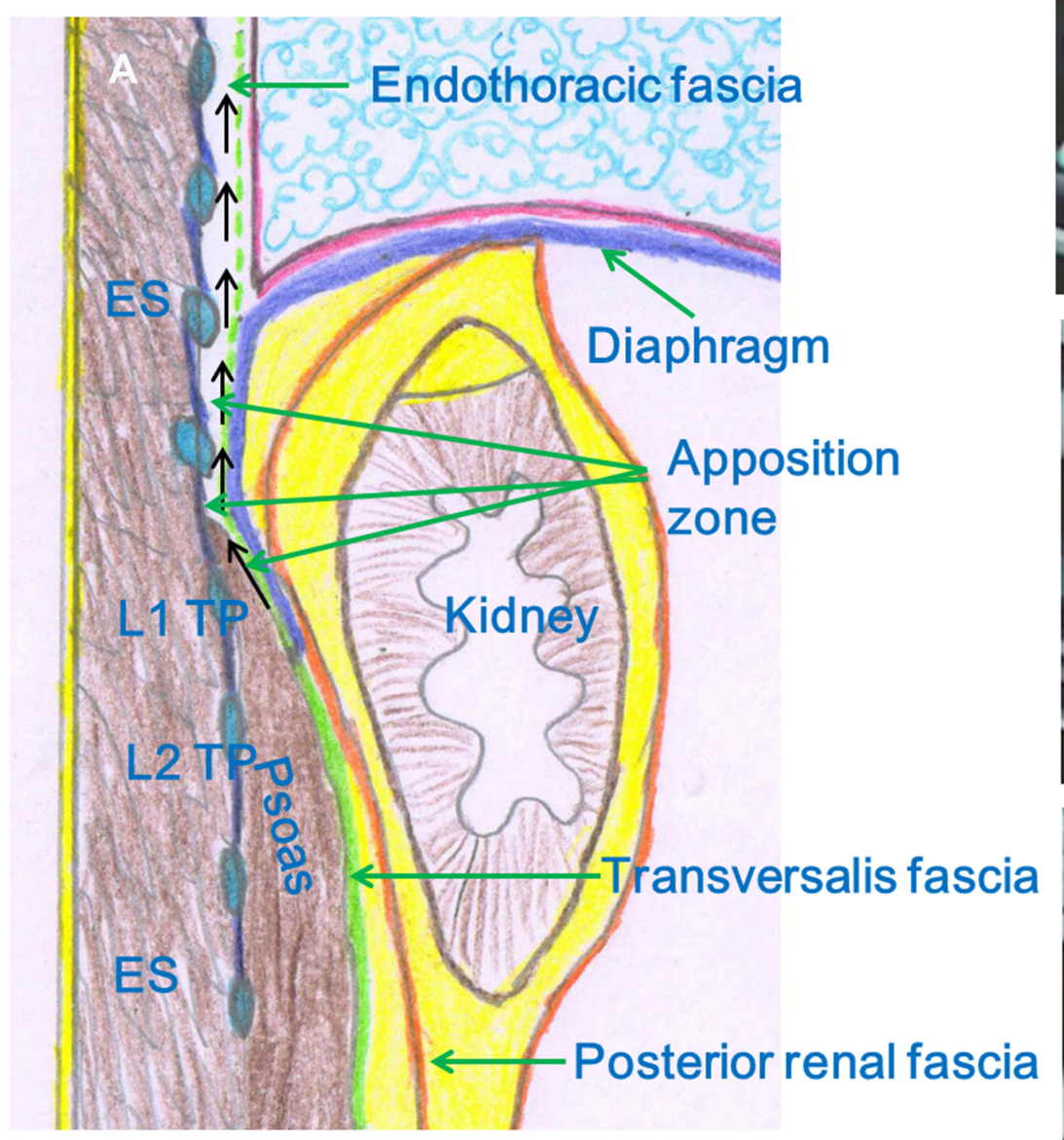

\section{Considerations}

Urologists should avoid damaging the diaphragm when dissecting the fascia tissue cephalad to look for the medial arcuate ligament. Otherwise, it can lead to disruption of the local anesthetic diffusion pathway, resulting in block failure. Prior to administration, the presence or absence of diaphragmatic injury should be evaluated. This technique should be avoided for patients with diaphragmatic injury. The ideal diffusion should be cephalic as well as medial and make the diaphragm bulge. Special attention should be paid to
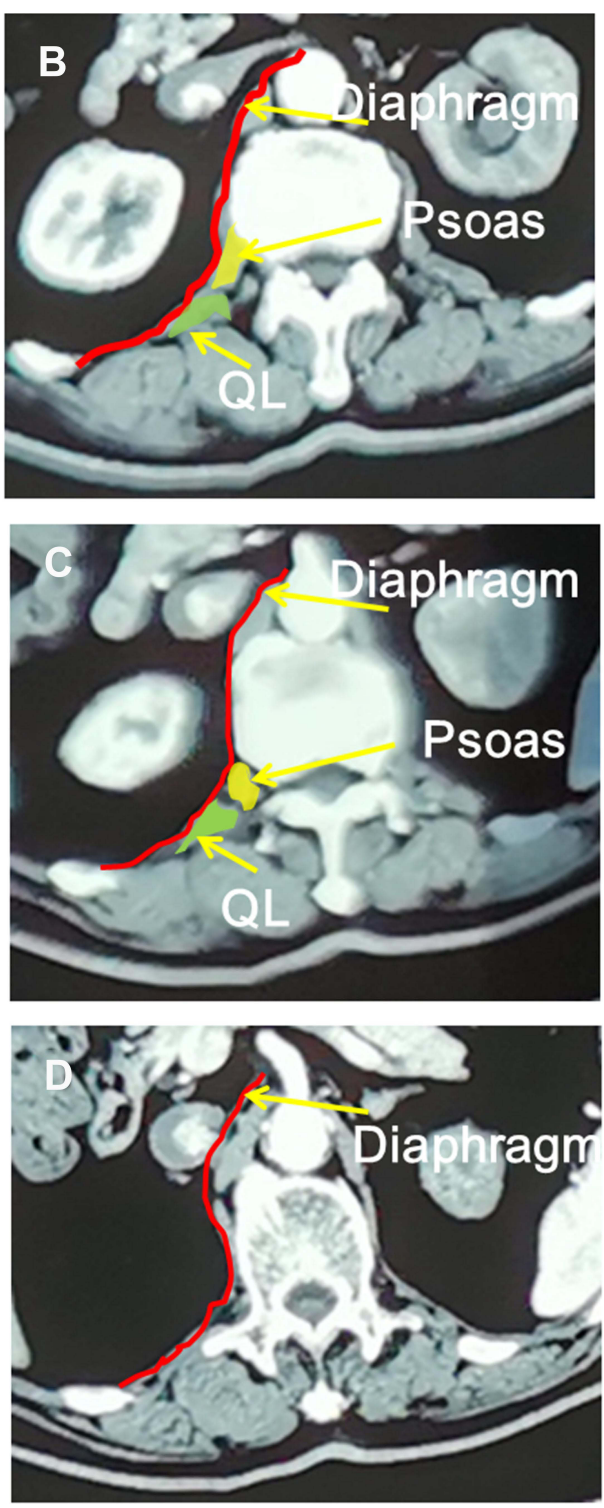

Figure 8 The communication relationship between the apposition zone of the diaphragm and lower thoracic paravertebral space. (A) The sagittal image at the level of midpoint of LI TP. The psoas fascia is in continuity with the endothoracic fascia (dashed green line) at the medial arcuate ligament of the diaphragm. The black arrow shows the LA spread from the apposition zone to the lower thoracic paravertebral space. (B-D) The computed tomography shows the cross-sections at the levels of lower edge of $L I$ vertebral body, upper edge of $L I$ vertebral body, and TI2 vertebral body, respectively. These images show that the apposition zone of the diaphragm at the level of $L I$ vertebral body gradually develops into the lower thoracic paravertebral space.

Abbreviations: TP, transverse process; LA, local anesthetic; ES, erector spinae; QL, quadratus lumborum. 
observe the diaphragmatic surface for liquid spills, which would suggest diaphragmatic injury or a superficial needle tip position. The apposition zone of the diaphragm is a potential compartment, and the resistance is minimal with $0.9 \%$ saline solution or local anesthetic injection. Great resistance when administering the drug suggests that the needle tip is not in the apposition zone, possibly within the diaphragm. If necessary, an appropriate amount of methylene blue can be added into the solution to visualize drug diffusion. Because of the intrinsic tension of the medial arcuate ligament and the direction of needle tip, the drug mainly diffuses cephalad and medially, which is a feature of this block. This feature has advantages over other techniques of anterior transmuscular QLB and anterior subcostal QLB. Retroperitoneal laparoscopic surgery frequently causes disruption of the transverse abdominal fascia, allowing leakage of the local anesthetic stored in fascial cavity. The dermatomal coverage of the sensory block is often between T6-7-L1-2 by ice cube method.

\section{Conclusions}

Clinical studies have found that supra-arcuate ligament blocks have rapid onset ( $<5$ minutes), long maintenance times (24-48 hours), and little effect on hemodynamic. ${ }^{5,22}$ Unilateral supra-arcuate ligament blocks can be used for surgical anesthesia and postoperative analgesia in lateral abdominal and retroperitoneal organ surgeries, while bilateral supra-arcuate ligament blocks can provide surgical anesthesia and postoperative analgesia for total abdominal surgery. ${ }^{25,26}$ Although a comprehensive understanding of a supra-arcuate ligament block has been obtained in clinical application, there is still much to learn about the drug diffusion pattern, which needs further cadaver or radiographic studies. Furthermore, there is also a need to strengthen comparative studies to investigate the prognostic impact of supra-arcuate ligament blocks over other nerve block techniques in surgical patients.

\section{Acknowledgments}

This work was supported by the Beijing Hospitals Authority Clinical Medicine Development of Special Funding Support (XMLX202106). All the patients have given consent to the publication of the images included in this review.

\section{Funding}

This work was supported by the Beijing Hospitals Authority Clinical Medicine Development of Special Funding Support (XMLX202106).

\section{Disclosure}

The authors declared no competing conflicts of interests.

\section{References}

1. Kadam VR. Ultrasound-guided quadratus lumborum block as a postoperative analgesic technique for laparotomy. J Anaesthesiol Clin Pharmacol. 2013;29:550-552. doi:10.4103/0970-9185.119148

2. Blanco R, Ansari T, Girgis E. Quadratus lumborum block for postoperative pain after caesarean section: a randomised controlled trial. Eur J Anaesthesiol. 2015;32:812-818. doi:10.1097/EJA.0000000000000299

3. Chakraborty A, Goswami J, Patro V. Ultrasound-guided continuous quadratus lumborum block for postoperative analgesia in a pediatric patient. A a Case Rep. 2015;4:34-36. doi:10.1213/XAA.0000000000000090

4. Murouchi T. Quadratus lumborum block intramuscular approach for pediatric surgery. Acta Anaesthesiol Taiwan. 2016;54:135-136. doi:10.1016/j.aat.2016.10.003

5. Li H, Ma D, Liu Y, Wang Y. A transverse approach for ultrasound-guided anterior quadratus lumborum block at the lateral supra-arcuate ligament. Anaesthesia. 2020;75:1400-1401. doi:10.1111/anae.15058

6. Blanco R. Tap block under ultrasound guidance: the description of a "no pops" technique: 271. Reg Anesth Pain Med. 2007;32:130. doi:10.1016/j.rapm.2006.11.012

7. Elsharkawy H. Quadratus lumborum block with paramedian sagittal oblique (subcostal) approach. Anaesthesia. 2016;71:241-242. doi:10.1111/anae.13371

8. Li H, Shi R, Wang Y. A modified approach below the lateral arcuate ligament to facilitate the subcostal anterior quadratus lumborum block. J Pain Res. 2021;14:961-967. doi:10.2147/JPR.S306696

9. Børglum J, Moriggl B, Jensen K. Ultrasound-guided transmuscular quadratus lumborum blockade. $B r \quad J$ Anesthesia. 2013;111:22. doi:10.1093/bja/el_9919

10. Carline L, Mcleod GA, Lamb C. A cadaver study comparing spread of dye and nerve involvement after three different quadratus lumborum blocks. Br J Anaesth. 2016;117:387-394. doi:10.1093/bja/ aew224

11. El-Boghdadly K, Elsharkawy H, Short A, Chin KJ. Quadratus lumborum block nomenclature and anatomical considerations. Reg Anesth Pain Med. 2016;41:548-549. doi:10.1097/AAP.0000000000000411

12. Elsharkawy H, El-Boghdadly K, Barrington M. Quadratus lumborum block: anatomical concepts, mechanisms, and techniques. Anesthesiology. 2019;130:322-335. doi:10.1097/ALN.00000000 00002524

13. Willard FH, Vleeming A, Schuenke MD, Danneels L, Schleip R. The thoracolumbar fascia: anatomy, function and clinical considerations. $J$ Anat. 2012;221:507-536. doi:10.1111/j.1469-7580.2012.01511.x

14. Dam M, Moriggl B, Hansen CK, Hoermann R, Bendtsen TF, Børglum J. The pathway of injectate spread with the transmuscular quadratus lumborum block: a cadaver study. Anesth Analg. 2017;125:303-312. doi:10.1213/ANE.0000000000001922

15. Elsharkawy H, El-Boghdadly K, Kolli S, et al. Injectate spread following anterior sub-costal and posterior approaches to the quadratus lumborum block: a comparative cadaveric study. Eur $J$ Anaesthesiol. 2017;34:587-595. doi:10.1097/EJA.0000000000000680

16. Tamura T, Yokota S, Ito S, Shibata Y, Nishiwaki K. Local anesthetic spread into the paravertebral space with two types of quadratus lumborum blocks: a crossover volunteer study. $J$ Anesth. 2019;33:26-32. doi:10.1007/s00540-018-2578-5

17. Alleyne CH Jr, Cawley CM, Shengelaia GG, Barrow DL. Microsurgical anatomy of the artery of Adamkiewicz and its segmental artery. $J$ Neurosurg. 1998;89:791-795. doi:10.3171/ jns.1998.89.5.0791

18. Kanemura T, Satake K, Nakashima H, et al. Understanding retroperitoneal anatomy for lateral approach spine surgery. Spine Surg Relat Res. 2017;1:107-120. doi:10.22603/ssrr.1.2017-0008 
19. Cai W, Li HZ, Zhang X, et al. Medial arcuate ligament: a new anatomic landmark facilitates the location of the renal artery in retroperitoneal laparoscopic renal surgery. J Endourol. 2013;27:64-67. doi:10.1089/end.2012.0152

20. Li H, Shi R, Wang Y. Use of transmuscular quadratus lumborum block for postoperative analgesia after laparoscopic nephrectomy. Reg Anesth Pain Med. 2021;46:1118-1119.

21. Li H, Shi R, Wang Y. Transincisional ultrasound-guided quadratus lumborum block in open renal surgeries. Pain Physician. 2021;24: E127-e8.

22. Shi R, Li H, Wang Y. Dermatomal coverage of single-injection ultrasound-guided parasagittal approach to anterior quadratus lumborum block at the lateral supra-arcuate ligament. $J$ Anesth. 2021;35:307-310. doi:10.1007/s00540-021-02903-1

23. Troyer AD, Wilson TA. Action of the diaphragm on the rib cage. $J$ Appl Physiol. 2016;121:391-400. doi:10.1152/japplphysiol.002 68.2016
24. Wu Z, Wang Y. Development of guidance techniques for regional anesthesia: past, present and future. J Pain Res. 2021;14:1631-1641. doi:10.2147/JPR.S316743

25. Huang X, Sun Y, Shi R, et al. Comparison of ultrasound-guided anterior quadratus lumborum block at the lateral supra-arcuate ligament with posterior quadratus lumborum block for perioperative analgesia in laparoscopic nephrectomy: a protocol for a randomised, prospective, parallel group, non-inferior trial. BMJ Open. 2021;11: e048490. doi:10.1136/bmjopen-2020-048490

26. Li H, Shi R, Shi D, Wang R, Liu Y, Wang Y. Anterior quadratus lumborum block at the lateral supra-arcuate ligament versus transmuscular quadratus lumborum block for postoperative analgesia in patients undergoing laparoscopic nephrectomy: a randomized controlled trial. J Clin Anesth. 2021;75:110561. doi:10.1016/j. jclinane.2021.110561

\section{Publish your work in this journal}

The Journal of Pain Research is an international, peer reviewed, open access, online journal that welcomes laboratory and clinical findings in the fields of pain research and the prevention and management of pain. Original research, reviews, symposium reports, hypothesis formation and commentaries are all considered for publication. The manuscript management system is completely online and includes a very quick and fair peer-review system, which is all easy to use. Visit http:// www.dovepress.com/testimonials.php to read real quotes from published authors. 